\title{
Redução da Velocidade de Cristalização a Frio do PET na Presença de Poliestireno
}

\author{
Renate M. R. Wellen \\ Engenharia de Processos, UFCG \\ Marcelo S. Rabello \\ Departamento de Engenharia de Materiais, UFCG
}

\begin{abstract}
Resumo: A cristalização a frio do poli(tereftalato de etileno) (PET) e de suas blendas com poliestireno (PS) foi estudada utilizando calorimetria exploratória diferencial (DSC), análise dinâmico-mecânica (DMA), microscopia eletrônica de varredura (MEV) e propriedades mecânicas. As blendas PET/PS formam misturas bifásicas, como confirmado por MEV e por DMA, onde fases distintas e duas $\mathrm{T}_{\mathrm{g}} \mathrm{s}$ foram observadas. Por outro lado, a determinação da temperatura de fusão de equilíbrio $\left(\mathrm{T}_{\mathrm{m}}^{\circ}\right)$ do PET por DSC indicou uma diminuição neste parâmetro com a presença do PS, sugerindo que uma solubilidade limitada do PS no PET deve ocorrer. A presença de um componente não cristalizável como o PS, parcialmente solúvel em uma fase cristalizável como PET, reduz a habilidade de cristalização. Isto foi observado nas análises de DSC através do deslocamento dos picos de cristalização a frio do PET para temperaturas maiores e por uma redução na velocidade de cristalização a frio. A abordagem de Avrami foi utilizada para os estudos cinéticos em condições isotérmicas, mostrando que o processo de cristalização ocorre em dois estágios e que a constante de cristalização K diminuiu significativamente com a presença de poliestireno. Nas blendas PET/PS a presença de apenas 1\% de PS retardou significantemente a cristalização do PET, em magnitude semelhante ao que ocorreu quando concentrações mais elevadas de PS foram utilizadas. Este comportamento é de grande importância industrial já que em baixa concentração de PS as propriedades mecânicas do PET não são afetadas, como também mostrado neste estudo.
\end{abstract}

Palavras-chave: Cristalização isotérmica a frio, PET, poliestireno, blendas.

\section{The Reduction of the Cold Crystallization Rate of PET in the Presence of Polystyrene}

Abstract: The cold crystallization of PET and its blends with polystyrene (PS) was investigated by differential scanning calorimetry (DSC), dynamic mechanical thermal analysis (DMA), scanning electron microscopy (SEM) and mechanical properties. PET/PS blends form separate phases, as confirmed by SEM and DMA, showing distinct phases and two glass transition temperatures. On the other hand, the determination of the equilibrium melting temperature of PET indicated that this parameter decreased when PS was added, suggesting that a limited solubility should exist. The presence of non crystallizable molecules like polystyrene, partially soluble in the PET crystallizable phase, reduces the driving force for crystallization. In the current study this effect was observed as a shifting of the cold crystallization DSC peaks to higher temperatures and also by the reduction in the rate of cold crystallization. The approach developed by Avrami was applied to study the kinetics of cold crystallization under isothermal conditions. It was shown that the crystallization occurs in two stages and that the rate constant K decreased significantly when PS was present. The blends containing only $1 \%$ of PS had the same magnitude of reduction in the rate of crystallization as the blends with higher PS content. This behaviour has a high practical significance since in low concentrations of PS the mechanical properties of PET are not affected, as also shown in this study.

Keywords: Isothermal cold crystallization, PET, polystyrene, blends.

\section{Introdução}

O poli (tereftalato de etileno) (PET) é atualmente um dos mais importantes termoplásticos de engenharia, podendo ser empregado na fabricação de uma grande variedade de produtos de embalagens como refrigerantes, águas, sucos e óleos comestíveis além de uma vasta gama de artigos injetados, termoformados e extrudados. A crescente partici- pação de PET no mercado de termoplásticos deve-se principalmente ao excelente balanço de propriedades mecânicas, térmicas e de barreira, aliadas a um custo relativamente baixo da matéria-prima.

OPET apresenta uma unidade química repetitiva composta de grupos de ácido tereftálico (grupo aromático), que formam o segmento rígido, e do etileno glicol (grupo alifático),

Autor para correspondência: Marcelo S. Rabello, Departamento de Engenharia de Materiais, UFCG, Av. Aprígio Veloso 882, CEP 58109-970, Campina Grande, PB, Brasil. E-mail: marcelo@dema.ufcg.edu.br. 
que é a estrutura flexível da cadeia polimérica. A estrutura molecular do PET é regular e apresenta polaridade média, fatores que favorecem a sua cristalização a partir do estado fundido. Entretanto, a presença do grupo aromático na cadeia principal reduz consideravelmente a velocidade de cristalização e, conseqüentemente, produtos amorfos são obtidos quando o material é resfriado rapidamente. Isso ocorre comumente em processos industriais, como na fabricação de pré-formas injetadas utilizadas na confecção de produtos soprados e em chapas e filmes extrudados. Em muitos casos (como em injeção-sopro e em termoformagem) o produto amorfo é submetido a um aquecimento (em temperaturas acima da temperatura vítrea do polímero, $\mathrm{T}_{\mathrm{g}}$ ) para que adquira a maleabilidade típica do estado borrachoso, permitindo a conformação na forma final. Se durante essa fase de reaquecimento a temperatura (ou o tempo) for inadequada pode-se inviabilizar a fase de conformação devido à cristalização a frio. Esse fenômeno ocorre acima da $T_{g}$, quando as moléculas do PET adquirem mobilidade suficiente para se rearranjarem em uma estrutura cristalina. Durante uma análise térmica por DSC a cristalização a frio é observada como um pico exotérmico entre a $T_{g}$ e a $T_{m}$, conforme ilustrado na Figura 1. Embora a cristalizaçã̃o a frio seja indesejável durante o reaquecimento da pré-forma (ou chapa), sua ocorrência durante a fase de conformação é importante, pois o material semicristalino possui melhores propriedades mecânicas e de barreira.

Apesar da importância tecnológica da cristalização a frio, a literatura científica dedicou relativamente pouca atenção a esse efeito no PET. Groeninckx et al. ${ }^{[1]}$ analisaram o desenvolvimento da estrutura morfológica do PET em função da temperatura de cristalização a frio $\left(\mathrm{T}_{\mathrm{c}}\right)$, verificando que em baixas $\mathrm{T}_{\mathrm{c}}$ 's lamelas altamente ramificadas com pequenas dimensões laterais foram formadas enquanto que em altas $T_{c}$ 's formou-se estruturas lamelares usuais. Pingping et al. ${ }^{[2]}$ registraram que a cristalização a frio do PET foi facilitada com a presença do $\mathrm{CaCO}_{3}$. Zhao et al. ${ }^{[3]} \mathrm{e} \mathrm{Lim} \mathrm{et} \mathrm{al.}{ }^{[4]}$ observaram um deslocamento do pico de cristalização a frio do PET para temperaturas inferiores quando o PET foi recozido em temperaturas próximas a $\mathrm{T}_{\mathrm{g}}$ e quando filamentos de PET foram estirados em

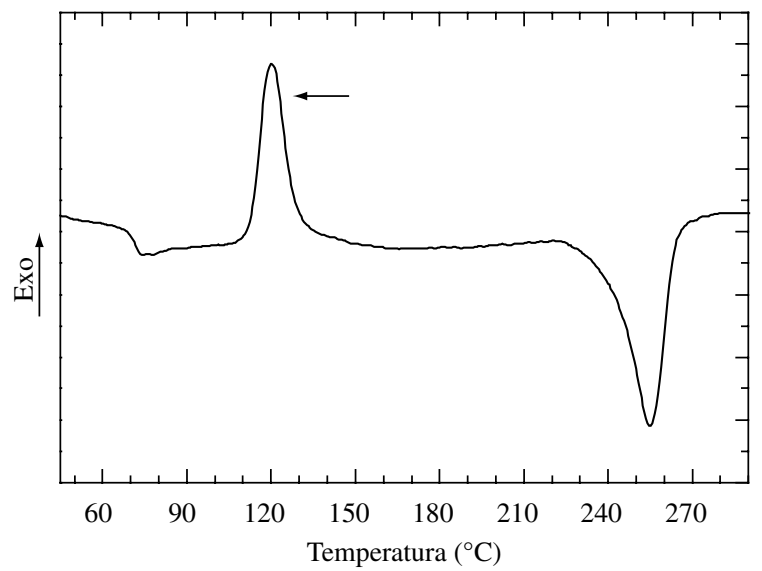

Figura 1. Curva de DSC do PET ilustrando a cristalização a frio (indicada pela seta). altas velocidades, respectivamente. Kint et al.$^{[5]}$ conseguiram diminuir a taxa de cristalização a frio do PET com a introdução de comonômeros em suas cadeias poliméricas. A utilização de grades de alto peso molecular ${ }^{[6,7]}$ como também a adição de diferentes polímeros como o policarbonato ${ }^{[8]} \mathrm{e}$ o poli(naftalato de etileno) (PEN) $)^{[9]}$ reduziram a velocidade de cristalização do PET. Em trabalho anterior ${ }^{[10]}$, os presentes autores avaliaram a cinética de cristalização a frio de chapas extrudadas de PET, observando que a velocidade de cristalização é fortemente dependente da temperatura e o comportamento mecânico do material é afetado pela estrutura cristalina obtida.

A necessidade de se deslocar a temperatura de cristalização a frio do PET para valores mais elevados e controlar a sua cinética de cristalização é um aspecto de grande importância tecnológica, pois reflete na redução do refugo industrial em fábricas de garrafas e de produtos termoformados. Embora algumas estratégias para se alcançar esse objetivo tenham sido tentadas, estas envolveram a incorporação de comonômeros não cristalizáveis na estrutura molecular do $\mathrm{PET}^{[11]}$. Embora viável, esta é uma solução complexa e pouco versátil uma vez que envolve alteração no processo de polimerização. Um procedimento mais simples de execução e de custo mais baixo pode representar um grande atrativo para a indústria.

A abordagem utilizada no presente trabalho foi a adição do poliestireno (PS) ao PET. Como um termoplástico não cristalizável, transparente, de boa processabilidade e de baixo custo, o poliestireno é muito utilizado em embalagens, principalmente em recipientes para a indústria alimentícia, descartáveis e outros utensílios domésticos. As blendas de PET com PS foram reportadas como bifásicas ${ }^{[12,13]}$, com suas propriedades dependendo do tamanho, da forma, da orientação e perfeição dos cristalitos, como também do grau de cristalinidade do PET. A adição do PS deve influenciar no mecanismo e cinética de cristalização a frio do PET, uma vez que representa imperfeições moleculares que podem perturbar a capacidade de ordenação cristalina do PET. Entretanto, o grau de interferência que o poliestireno pode provocar depende de fatores como a miscibilidade com o PET e condições de cristalização. $O$ presente trabalho investiga o efeito da adição de poliestireno no fenômeno da cristalização a frio no PET, com ênfase na cinética de cristalização e nas temperaturas de transições térmicas. O objetivo mais global do estudo é viabilizar um procedimento simples e eficaz para redução do percentual de defeitos resultante da cristalização prematura do PET nas indústrias de transformação sem afetar suas outras características como propriedades óticas e mecânicas.

\section{Experimental}

\section{Materiais}

O PET usado neste trabalho foi o Rhopet $\mathrm{S} 78$ produzido pela Rhodia, grau garrafa, apresentando uma viscosidade intrínseca de $0,78 \mathrm{dL} \cdot \mathrm{g}^{-1}, \bar{M}_{w}=48.000 \mathrm{~g} \cdot \mathrm{mol}^{-1}$. O PS foi o Dow Styron 649D usado em moldagem por injeção. 


\section{Preparação das blendas}

Antes do processo de mistura o PET foi seco em estufa com circulação forçada de ar a $120{ }^{\circ} \mathrm{C}$ por 6 horas para evitar a hidrólise durante o processamento ${ }^{[14]}$ enquanto que o poliestireno foi seco a $80^{\circ} \mathrm{C}$ por 14 horas. A mistura foi realizada no misturador interno de um reômetro tipo Haake por 10 minutos a uma temperatura $265{ }^{\circ} \mathrm{C}$ e velocidade dos rotores de $60 \mathrm{rpm}$. Para todas as composições foram utilizados $50 \mathrm{~g}$ de material. Após a mistura o "melt" foi rapidamente resfriado em água/gelo para evitar cristalização do PET. As blendas foram preparadas com as seguintes composições (em massa) de PS: $1,2,5,10,15,20$ e $30 \%$.

\section{DSC e cinética de cristalização}

A análise térmica por calorimetria exploratória diferencial (DSC) foi conduzida em um equipamento Shimadzu DSC-50 utilizando-se 5-7 mg de amostra e uma velocidade de aquecimento de $10{ }^{\circ} \mathrm{C} \cdot \mathrm{min}^{-1}$. Os termogramas aqui reportados foram obtidos apenas durante a primeira varredura de aquecimento. A calibração do equipamento foi realizada com índio, e a correção da linha base foi feita da temperatura ambiente até $300{ }^{\circ} \mathrm{C}$. Para cada composição analisada foi realizada uma nova calibração e uma correção da linha base, minimizando erros com respeito à determinação dos tempos de início e término da cristalização ${ }^{[15]}$.

O estudo da cinética de cristalização foi realizado sob condições isotérmicas através de um rápido aquecimento $\left(\approx 100^{\circ} \mathrm{C} \cdot \mathrm{min}^{-1}\right)$ da temperatura ambiente até a temperatura alvo de cristalização isotérmica seguindo-se procedimentos anteriores ${ }^{[10]}$. A exoterma de cristalização foi registrada em função do tempo até que nenhuma variação com respeito à linha base fosse observada. As temperaturas de cristalização variaram entre 100 e $160{ }^{\circ} \mathrm{C}$, com intervalos de $5{ }^{\circ} \mathrm{C}$. Para cada temperatura foram realizados 2 ensaios. A partir dos termogramas dados cinéticos como velocidade de cristalização e curva de Avrami foram calculados de acordo com o procedimento descrito anteriormente ${ }^{[10]}$.

\section{Análise dinâmico-mecânica (DMA)}

As análises de DMA foram realizadas no equipamento DMA 983 da TA Instruments, e as curvas foram obtidas por aquecimento das amostras amorfas no intervalo de temperatura entre 0 e $200{ }^{\circ} \mathrm{C}$, numa frequêencia de $1 \mathrm{~Hz}$ e taxa de aquecimento de $2{ }^{\circ} \mathrm{C} \cdot \mathrm{min}^{-1}$, sob fluxo de nitrogênio.

\section{Difração de raios $X$}

Os difratogramas de raios $X$ foram obtidos em um aparelho XRD 6000 Shimadzu, numa faixa de $2 \theta=0$ a $40^{\circ}$, a uma velocidade de $2^{\circ} \cdot \mathrm{min}^{-1}$, tensão de $40 \mathrm{Kv}$ e amperagem de $30 \mathrm{~mA}$, com fendas de 0,15 e $0,5 \mathrm{~mm}$.

\section{Microscopia eletrônica de varredura (MEV)}

Análises de microscopia eletrônica de varredura (MEV) das superfícies de fratura foram realizadas em equipamen- to SSX 550 Superscan da Shimadzu após recobrimento com ouro para evitar acúmulo de carga estática. As amostras foram previamente fraturadas em nitrogênio líquido e, assim, a deformação plástica foi evitada.

\section{Confecção de corpos de prova e propriedades mecânicas}

Foi analisado o comportamento mecânico do PET e da blenda com $1 \%$ de PS. Os corpos de prova foram obtidos por injeção após mistura em extrusora. A mistura ocorreu em extrusora dupla rosca Extruder TW 100 a $260{ }^{\circ} \mathrm{C}$ (todas as zonas) e velocidade das roscas de $60 \mathrm{rpm}$. O material triturado foi então seco em estufa a $100{ }^{\circ} \mathrm{C}$ por 4 horas e corpos de prova para ensaios de tração foram obtidos numa Injetora Fluidmec H30-40. Utilizou-se uma temperatura de $260{ }^{\circ} \mathrm{C}$ (cilindro e bico) e tempo de resfriamento de 25 segundos. $\mathrm{O}$ molde foi resfriado com circulação de água gelada $\left(\sim 10^{\circ} \mathrm{C}\right)$ para evitar a cristalização durante o resfriamento. Alguns corpos de prova foram submetidos a um tratamento térmico em estufa por 1 hora a $150{ }^{\circ} \mathrm{C}$ para a ocorrência da cristalização a frio.

Para cada composição analisada 2 corpos de prova injetados foram selecionados e submetidos ao ensaio de difração de raios X para confirmar a ausência ou presença de cristalinidade.

Os ensaios mecânicos dos corpos de prova foram realizados utilizando-se um equipamento de ensaios mecânicos LLOYD Instruments LR10K, a $23{ }^{\circ} \mathrm{C}$, operando a uma velocidade de estiramento de $5 \mathrm{~mm} \cdot \mathrm{min}^{-1}$. Os resultados foram analisados segundo a norma D 638 da ASTM e os dados reportados representam médias de 6 amostras.

\section{Resultados e Discussão}

\section{Análise térmica por DSC}

As curvas de DSC obtidas durante o aquecimento do PET e das blendas estão mostradas na Figura 2 e as temperaturas e calores associados com os processos de cristalização a frio e de fusão estão resumidos na Tabela 1 . Ob-

Tabela 1. Temperaturas e entalpias associadas com os processos de cristalização a frio e fusão dos materiais estudados.

\begin{tabular}{lcccccc}
\hline Composição & $\begin{array}{c}\mathbf{T}_{\mathbf{c i}} \\
\left({ }^{\circ} \mathbf{C}\right)\end{array}$ & $\begin{array}{c}\mathbf{T}_{\mathbf{c}} \\
\left({ }^{\mathbf{}} \mathbf{C}\right)\end{array}$ & $\begin{array}{c}\mathbf{T}_{\mathbf{c f}} \\
\left({ }^{\mathbf{0}} \mathbf{C}\right)\end{array}$ & $\begin{array}{c}\Delta \mathbf{H}_{\mathbf{c}} \\
(\mathbf{J} / \mathbf{g})\end{array}$ & $\begin{array}{c}\mathbf{T}_{\mathbf{m}} \\
\left({ }^{\mathbf{o}} \mathbf{C}\right)\end{array}$ & $\begin{array}{c}\Delta \mathbf{H}_{\mathbf{m}} \\
(\mathbf{J} / \mathbf{g})\end{array}$ \\
\hline PET & 113,0 & 124,4 & 139,4 & 20,9 & 248,7 & 29,5 \\
1\%PS & 113,4 & 140,6 & 155,6 & 18,2 & 249,1 & 28,4 \\
2\%PS & 116,8 & 136,3 & 159,8 & 20,8 & 248,2 & 29,8 \\
5\%PS & 116,3 & 146,5 & 162,6 & 21,1 & 248,0 & 29,9 \\
10\%PS & 119,4 & 146,9 & 161,6 & 18,9 & 248,0 & 29,2 \\
15\%PS & 112,3 & 144,2 & 159,7 & 16,4 & 250,5 & 27,5 \\
20\%PS & 117,6 & 143,1 & 160,1 & 19,2 & 247,1 & 32,5 \\
30\%PS & 122,4 & 140,7 & 158,3 & 17,4 & 247,5 & 33,7 \\
\hline
\end{tabular}

$\mathrm{T}_{\mathrm{ci}}, \mathrm{T}_{\mathrm{c}}$ e $\mathrm{T}_{\mathrm{cf}}$ são as temperaturas do início, do pico e do término da cristalização a frio, respectivamente; $\mathrm{T}_{\mathrm{m}}$ é a temperatura de fusão; e $\Delta \mathrm{H}_{\mathrm{c}} \mathrm{e} \Delta \mathrm{H}_{\mathrm{m}}$ são as entalpias de cristalização a frio e fusão, respectivamente, corrigidas para o teor de PET. 

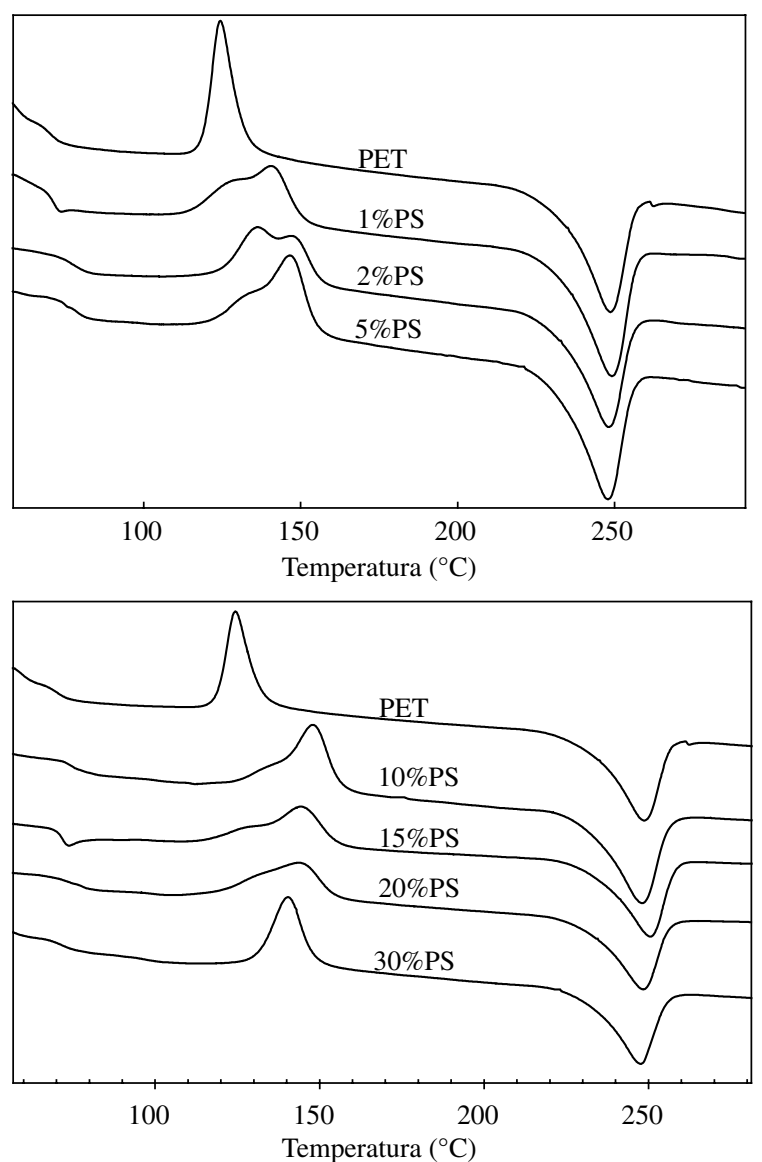

Figura 2. Curvas de DSC das amostras PET e blendas com diferentes teores de poliestireno.

serva-se um deslocamento do pico de cristalização a frio do PET para temperaturas mais elevadas com a adição do PS, o que vai ao encontro dos objetivos iniciais deste estudo. A cristalização a frio no PET foi observada ocorrer em $\approx 124$ ${ }^{\circ} \mathrm{C}$, e nas blendas com PS este valor foi deslocado para até $\approx 147{ }^{\circ} \mathrm{C}$, em variações não sistemáticas. Trata-se de uma alteração significativa que, certamente, representa grandes impactos na facilidade de processamento deste material. Observa-se também que a adição de PS em quantidades superiores a $1 \%$ não promove modificações substanciais na faixa de temperatura onde ocorre a cristalização a frio do PET. Esse dado é altamente relevante, pois em concentração tão baixa, espera-se efeito mínimo nas demais propriedades do PET (ver adiante os dados de propriedades mecânicas). O comportamento de fusão do PET sofreu apenas pequenas oscilações com a presença de PS, com picos de fusão apresentando valores próximos a $250{ }^{\circ} \mathrm{C} \mathrm{em}$ todas as amostras. Esse fato sugere que o poliestireno afeta a cristalização a frio do PET, mas não interfere na sua estrutura cristalina. Da mesma forma, as entalpias de fusão e de cristalização mantiveram-se aproximadamente invariáveis. Tomando como base os resultados mostrados na Figura 2 as composições com 1 e $15 \%$ de PS foram escolhidas para reportar os parâmetros cinéticos.

\section{Termogramas de DMA e estudos de miscibilidade}

Um dos critérios mais comuns para se avaliar a miscibilidade de blendas é a análise das temperaturas vítreas dos componentes em uma mistura. Quando as $\mathrm{T}_{\mathrm{g}}$ 's dos componentes das blendas não são deslocadas em relação aos valores originais a blenda é considerada imiscível enquanto que a obtenção de uma única $\mathrm{T}_{\mathrm{g}}$ em valor intermediário denota miscibilidade $^{[16]}$. Nas curvas de DSC mostradas na Figura 2 só foi possível a visualização da $\mathrm{T}_{\mathrm{g}}$ do PET em posição equivalente à $\mathrm{T}_{\mathrm{g}}$ do PET puro. Como não houve deslocamento desta tem-

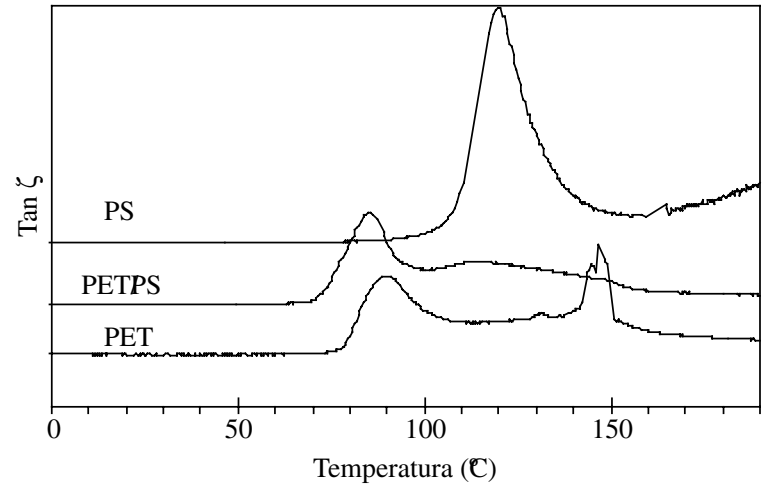

Figura 3. DMA das amostras PS, PET e blenda com 15\% de PS.

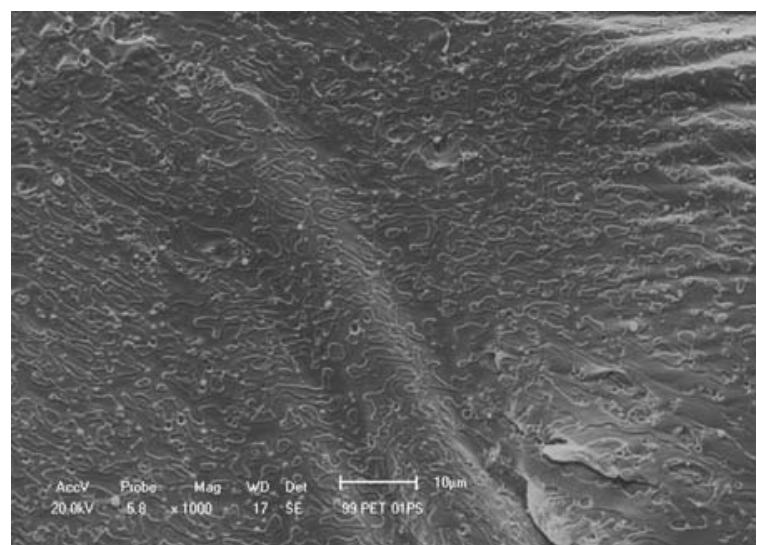

(a)

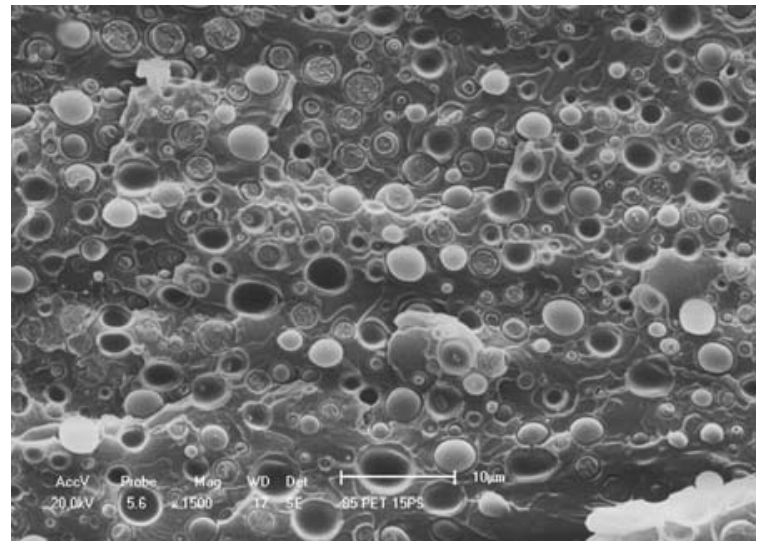

(b)

Figura 4. Microscopia eletrônica da superfície de fratura de blendas de PET a) com $1 \%$; e b) com $15 \%$ de poliestireno. A barra de escala equivale a $10 \mu \mathrm{m}$. 
peratura de transição assim como a $T_{g}$ do PS não foi detectada, inferiu-se que a técnica de DSC não foi suficientemente sensível para se concluir sobre a miscibilidade da mistura. As amostras foram então submetidas a análises de DMA, que é uma técnica mais sensível para a determinação de $\mathrm{T}_{\mathrm{g}}$. As curvas de DMA do PS, do PET e da blenda com $15 \%$ de PS estão ilustradas na Figura 3, onde é possível observar na cur-

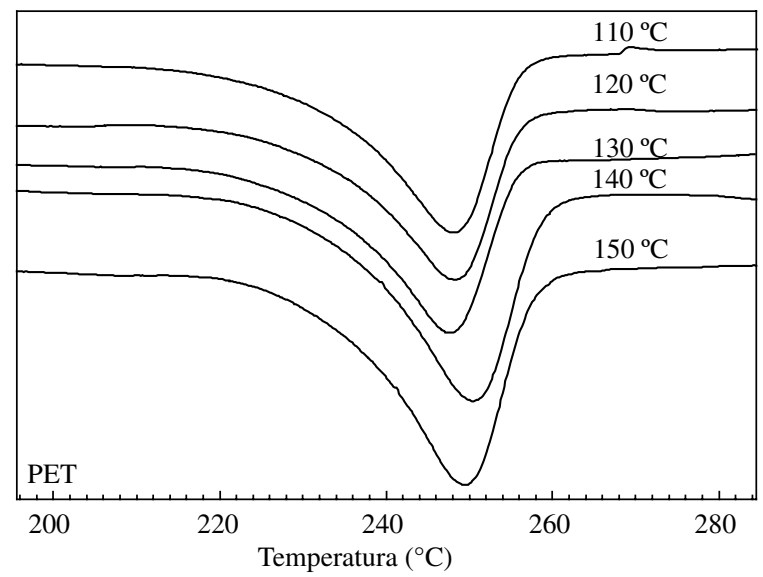

(a)

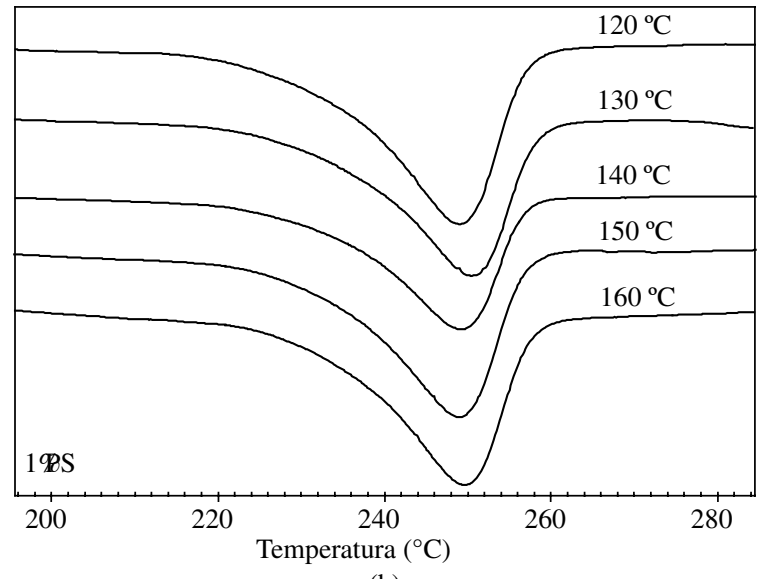

(b)

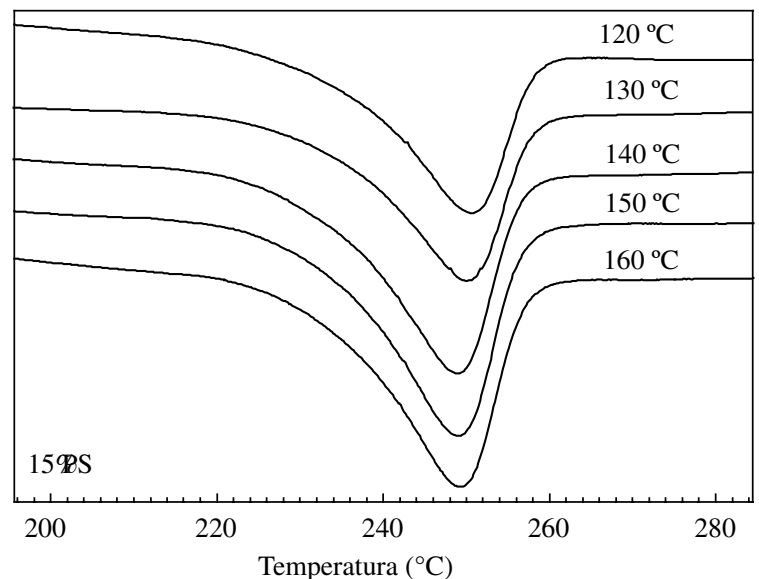

(c)

Figura 5. Endotermas de fusão do a) PET; b) blendas com 1\%; e c) $15 \%$ de PS cristalizadas isotermicamente a frio em diferentes temperaturas. va da blenda que as $\mathrm{T}_{\mathrm{g}} \mathrm{s}$ do PET e do PS situam-se próximas dos valores originais, indicando que os polímeros formam misturas bifásicas.

As análises de microscopia eletrônica de varredura das superfícies de fratura das blendas estão mostradas na Figura 4. Nas duas imagens a separação de fases é muito evidente, com as partículas esféricas de PS dispersas na matriz de PET. $\mathrm{Na}$ blenda com $1 \%$ de PS as partículas apresentam diâmetros $\approx 0,8 \mu \mathrm{m}$ e na blenda com $15 \%$ tem-se partículas com $\approx 2,6 \mu \mathrm{m}$ de diâmetro. Nota-se também nas micrografias que os polímeros PET e PS apresentam baixa adesão interfacial, já que partículas de PS foram sacadas da matriz PET.

A miscibilidade das blendas obtidas pela adição de um componente amorfo a um polímero cristalizável também pode ser avaliada pela depressão da temperatura de fusão. Em sistemas miscíveis a temperatura de fusão da blenda será mais baixa do que a do componente cristalizável puro, como resultado de fatores cinéticos, morfológicos e termodinâmicos, que contribuem para a formação de cristais menos estáveis $^{[17]}$. Estudo do comportamento de fusão foi então realizado nas amostras cristalizadas isotermicamente a partir do estado sólido em diferentes temperaturas. A Figura 5 mostra endotermas de fusão do PET e das blendas com $1 \%$ e com $15 \%$ de PS submetidas à cristalização isotérmica nas temperaturas indicadas. Observa-se que, aparentemente, o comportamento de fusão do PET não alterou de forma significativa com a adição do PS e com as diferentes temperaturas de cristalização. Como a fusão ocorre em uma ampla faixa de temperaturas em virtude de diferentes tamanhos e graus de perfeição dos cristais presentes e a $\mathrm{T}_{\mathrm{m}}$ é determinada como o pico da curva, o seu valor não representa verdadeiramente uma propriedade intrínseca do material. Para isso, determina-se geralmente a temperatura de fusão de equilíbrio $\left(\mathrm{T}_{\mathrm{m}}^{\mathrm{o}}\right)$ que representa a estabilidade de cristais de tamanho infinito ${ }^{[18]} \mathrm{e}$, assim, se constitui em um melhor parâmetro para avaliar as diferenças de estabilidade térmica das várias composições. A partir dessas endotermas a temperatura de fusão de equilíbrio foi calculada segundo o método proposto por Hoffman e Weeks ${ }^{[19]}$, por extrapolação da condição $\mathrm{T}_{\mathrm{m}}=\mathrm{T}_{\mathrm{c}}$ utilizando-se amostras cristalizadas em várias temperaturas. $\mathrm{O}$ PET apresentou uma $\mathrm{T}_{\mathrm{m}}^{\mathrm{b}} \approx 255^{\circ} \mathrm{C}$, a qual está dentro da faixa de valores encontrados na literatura ${ }^{[1,6]}$. Os valores obtidos para as blendas com $1 \%$ e com $15 \%$ foram $\sim 250$ e $\sim 246^{\circ} \mathrm{C}$, respectivamente. Observa-se que ocorreu um pequeno (mas consistente) decréscimo na temperatura de fusão de equilíbrio do PET com a presença do poliestireno. Esses resultados sugerem que, ao contrário das observações feitas por DMA, as blendas PET/ PS não podem ser consideradas como completamente imiscíveis já que depressões na $\mathrm{T}_{\mathrm{m}}^{\mathrm{b}}$ foram verificadas.

\section{Cinética de cristalização isotérmica a frio}

Algumas curvas de DSC obtidas durante a cristalização isotérmica a frio do PET e das blendas estão mostradas na Figura 6. Nas amostras analisadas um aumento na temperatura de cristalização promoveu um deslocamento das exotermas 


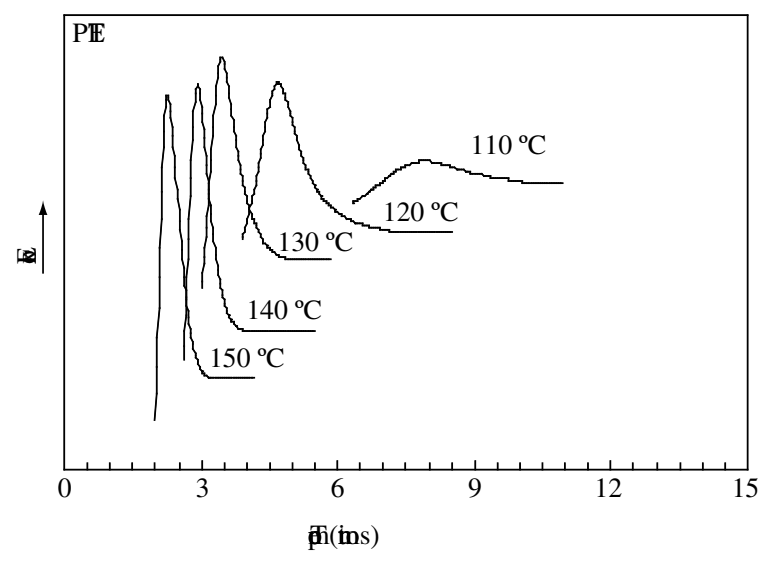

(a)

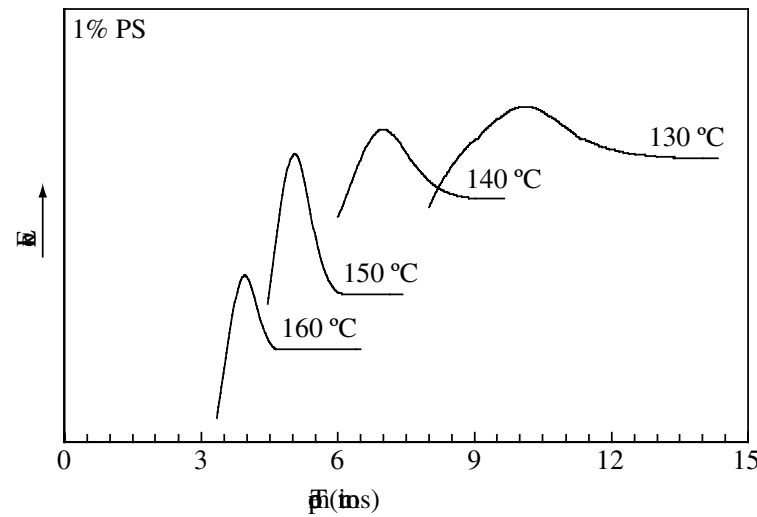

(b)

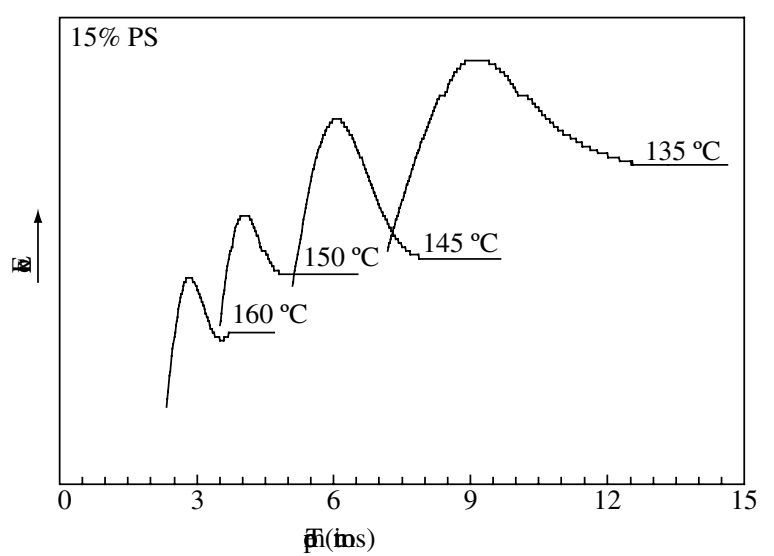

(c)

Figura 6. Exemplos de exotermas de DSC obtidas durante a cristalização isotérmica a frio do PET e da blenda com 1 e $15 \%$ de poliestireno (temperaturas de cristalização a frio indicadas).

para tempos menores, evidenciando um aumento na velocidade de cristalização. Isso é conseqüência de uma maior mobilidade molecular, o que facilita a ordenação cristalina. Analisando o comportamento de cristalização das três amostras numa dada temperatura, observa-se que a cristalização a frio no PET ocorreu em um menor intervalo de tempo em relação ao das blendas com PS. Como já mencionado, as moléculas atáticas do poliestireno afetam o comportamento do componente cristalizável, pois representa impurezas que reduzem a cristalizabilidade do PET $^{[20,21]}$.
Comportamento de certa forma similar ao observado nas blendas PET/PS foi previamente verificado durante a cristalização a partir do "melt" do polipropileno com diferentes tipos e quantidades de PP atático ${ }^{[20-23]}$. Nesses estudos foi observado que a cristalização do PP foi retardada com a adição das moléculas atáticas, que representam impurezas para a cristalização do PP. A segregação molecular das impurezas foi observada ocorrer entre os esferulitos e nas regiões interfibrilares, que depende de fatores como mobilidade das impurezas e condições de cristalização.

No presente estudo a redução na velocidade de cristalização do PET na presença do poliestireno pode estar associada com a solubilidade entre os dois polímeros. Embora o PET e o poliestireno sejam essencialmente imiscíveis (como mostrado anteriormente), é possível que haja uma pequena fração de moléculas de PS solúvel na fase amorfa do PET. Essa consideração baseia-se no próprio conceito de solubilidade, onde traços de um componente podem estar solúveis em um meio sem que isso caracterize uma mistura homogênea (quando o soluto está presente em maiores proporções). No caso da mistura PET/PS, o limite de solubilidade do poliestireno no PET deve ser baixo (certamente menos de $1 \%$, como verificado na Figura 4) mas mesmo assim pode dificultar a cristalização do PET pelo mesmo conceito de impurezas em polímeros cristalizáveis mencionado acima. A existência de um baixo limite de solubilidade explica a pouca influência da concentração de poliestireno na cinética de cristalização do PET, ou seja, atingido o limite de solubilidade, a presença de moléculas adicionais de poliestireno segregam para regiões fora da zona de cristalização e deixam de interferir na cristalizabilidade do PET.

A partir das exotermas de DSC mostradas na Figura 6 o desenvolvimento da cristalinidade com o tempo, $\mathrm{X}_{\mathrm{t}}$, foi calculado e as curvas obtidas estão mostradas na Figura 7. Todas as isotermas apresentaram uma forma sigmoidal caracterizando um processo de transformação de fases sem descontinuidades. As isotermas apresentando formas similares podem ser deslocadas possibilitando a superposição das curvas nas diferentes temperaturas. O desenvolvimento da cristalinidade nas blendas é mais lento e as isotermas só foram observadas em maiores intervalos de tempo, indicando que o PS retarda a cristalização do PET. Nas três amostras a cristalização prossegue a uma taxa acelerada, até ser atingido um estado de pseudo-equilíbrio, porém uma cristalização a taxas muito lentas continua ocorrendo por um período de tempo finito. Esse comportamento é atribuído à interferência entre cristais no final da cristalização primária que por razões estéricas reduz a velocidade de cristalização. Normalmente isto ocorre em regiões inter-esferulíticas de cristalinidade mais difícil e o processo é denominado de cristalização secundária ${ }^{[1,24]}$.

A cinética de cristalização isotérmica a frio foi analisada segundo a abordagem de Avrami ${ }^{[25-27]}$ e as curvas características foram construídas a partir das isotermas da Figura 7 e estão mostradas na Figura 8. Observa-se que algumas curvas apresentaram desvio de linearidade no final do processo de 


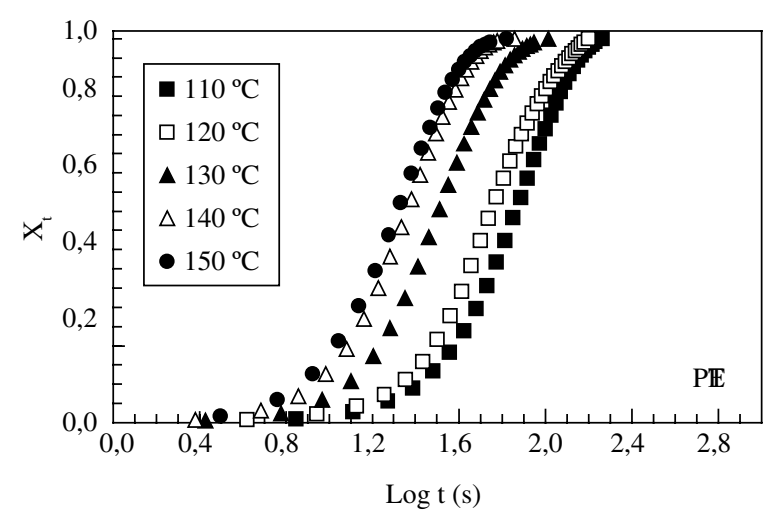

(a)

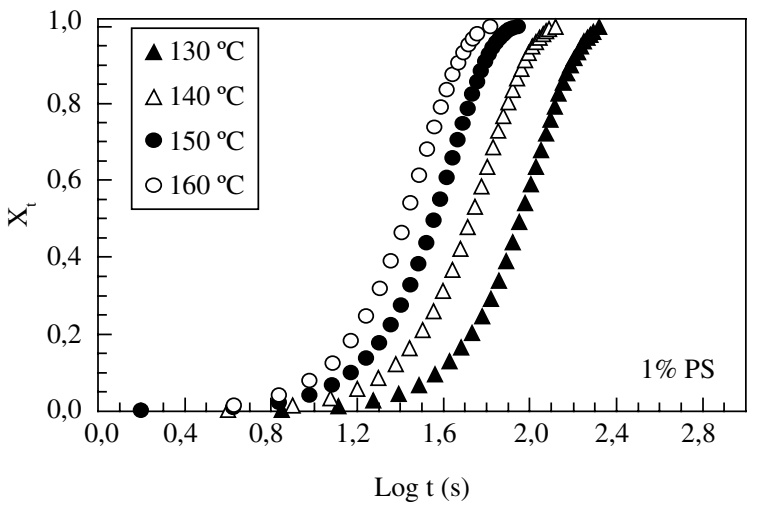

(b)

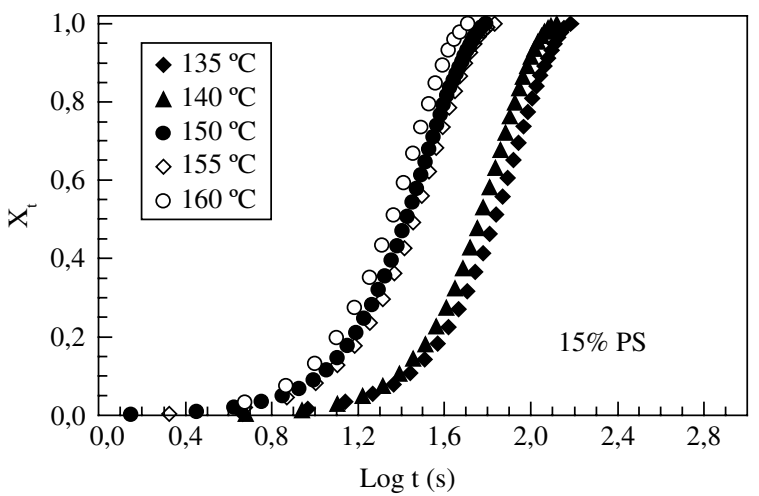

(c)

Figura 7. Desenvolvimento da cristalinidade com o tempo para o PET e para as blendas em diferentes temperaturas de cristalização a frio.

cristalização o que pode estar associado à cristalização secundária. A segregação de moléculas não cristalizáveis de PS contribui com a diminuição da taxa de crescimento cristalino podendo também estar relacionado com o desvio da linearidade nas curvas de Avrami durante a cristalização secundária $^{[20-21,28-29]}$

Os parâmetros de Avrami, n e K, foram calculados para porção linear ( $1^{\circ}$ estágio da cristalização) das curvas mostradas na Figura 8 e os resultados obtidos estão mostrados na Tabela 2. $\mathrm{O}$ expoente $\mathrm{n}$ apresentou valores próximos a 2 tanto para o PET quanto para as blendas com $1 \%$ e $15 \%$ de PS, valor associado a um crescimento em forma de disco gerado por nucleação heterogênea ${ }^{[25-27]}$. A constante de

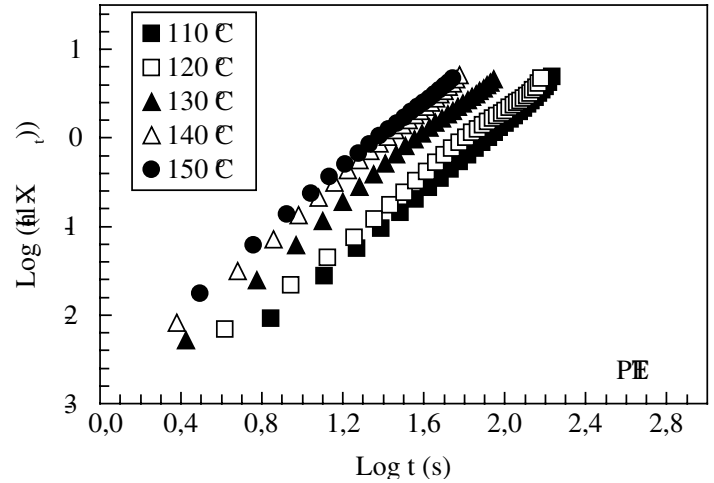

(a)

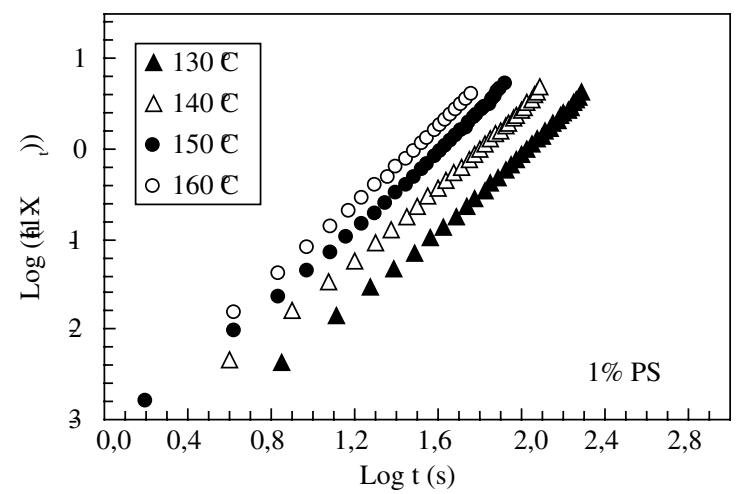

(b)

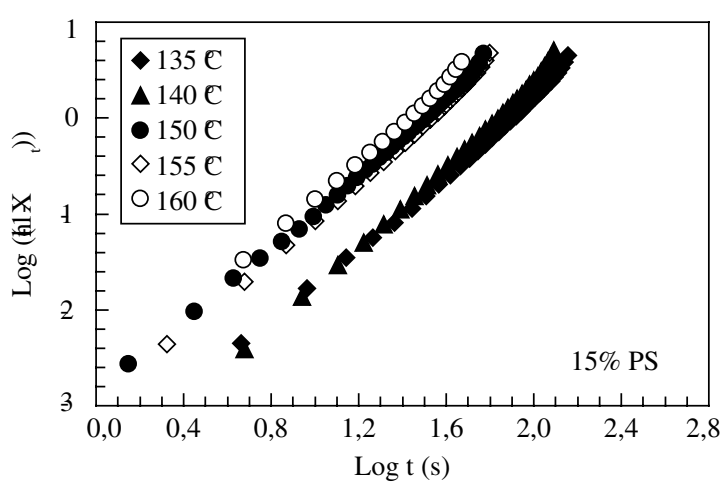

(c)

Figura 8. Curvas de Avrami para cristalização isotérmica a frio do PET e das blendas.

velocidade $\mathrm{K}$ aumentou com a temperatura de cristalização nos três sistemas, mas para as blendas com PS K apresentou valores bem inferiores em relação ao PET puro. Para citar um exemplo, quando as amostras foram submetidas à cristalização isotérmica a $150{ }^{\circ} \mathrm{C}$, o PET apresentou um valor para $\mathrm{K}$ de $2,317\left(10^{-3} \cdot \mathrm{s}^{-1}\right)$ enquanto que as blendas com $1 \%$ e $15 \%$ de PS apresentaram valores para K de 0,526 e 1,189 $\left(10^{-3} \cdot \mathrm{s}^{-1}\right)$, respectivamente. Neste caso, observou-se uma diminuição na velocidade de cristalização do PET de $\approx 80 \%$ na blenda com $1 \%$ de PS.

A influência da temperatura de cristalização e da adição de PS em K pode ser melhor observada nas curvas da Figura 9, onde nota-se claramente que as blendas apresentam 
Tabela 2. Parâmetros de Avrami obtidos para algumas temperaturas selecionadas. Nas curvas que apresentaram cristalização em 2 estágios, os valores foram calculados para o primeiro estágio.

\begin{tabular}{|c|c|c|c|c|c|c|}
\hline \multirow{2}{*}{$\begin{array}{c}\mathbf{T}_{\mathbf{c}} \\
\left({ }^{\circ} \mathbf{C}\right)\end{array}$} & \multicolumn{2}{|c|}{ PET } & \multicolumn{2}{|c|}{$1 \%$ PS } & \multicolumn{2}{|c|}{$15 \%$ PS } \\
\hline & $\mathrm{n}$ & $\mathrm{K}\left(10^{-3} \mathrm{~s}^{-1}\right)$ & $\mathrm{n}$ & $\mathrm{K}\left(10^{-3} \mathrm{~s}^{-1}\right)$ & $\mathrm{n}$ & $\mathrm{K}\left(10^{-3} \mathrm{~s}^{-1}\right)$ \\
\hline 130 & 2,00 & 0,732 & 2,02 & 0,076 & - & - \\
\hline 135 & 1,97 & 1,091 & 1,96 & 0,160 & 1,88 & 0,241 \\
\hline 140 & 1,98 & 1,518 & 1,99 & 0,250 & 2,10 & 0,137 \\
\hline 145 & 1,87 & 2,316 & 2,10 & 0,226 & 2,02 & 0,189 \\
\hline 150 & 1,90 & 2,317 & 1,99 & 0,526 & 1,95 & 1,189 \\
\hline
\end{tabular}

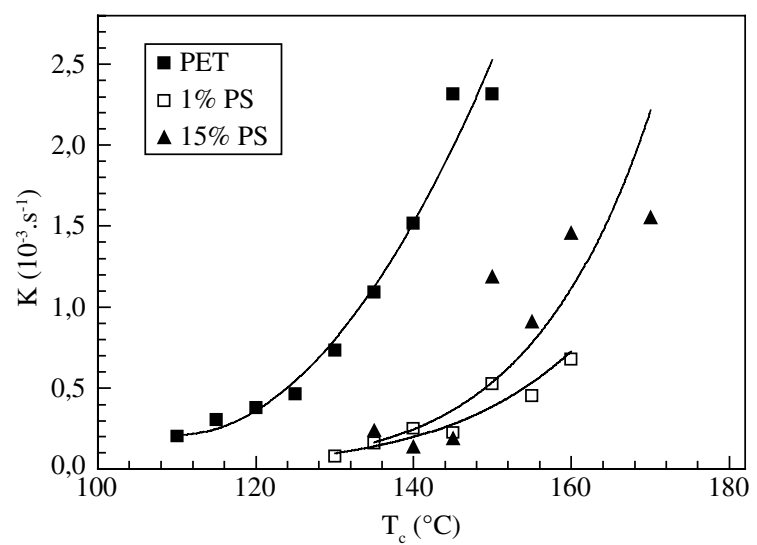

Figura 9. Efeito da temperatura de cristalização na constante de velocidade $\mathrm{K}$ do PET e das blendas.

uma cristalização bem mais lenta em relação ao polímero puro, indicando que o PS retardou consideravelmente a sua cristalização. Nota-se também que houve pouca diferença de comportamento entre as duas blendas. Esse resultado é altamente significativo em termos práticos, uma vez que a adição de apenas $1 \%$ de poliestireno, que provoca praticamente os mesmos efeitos da adição de $15 \%$ de PS, deve provocar efeitos mínimos nas propriedades mecânicas, propriedades óticas e processabilidade do PET.

A diminuição da taxa de cristalização do PET foi previamente observada em diferentes sistemas poliméricos como é o caso das blendas PET/PC ${ }^{[8]}$, PET/PEI ${ }^{[30]}$ e PET/PVF ${ }_{2}^{[31]}$, como resultado dos processos de segregação de moléculas do

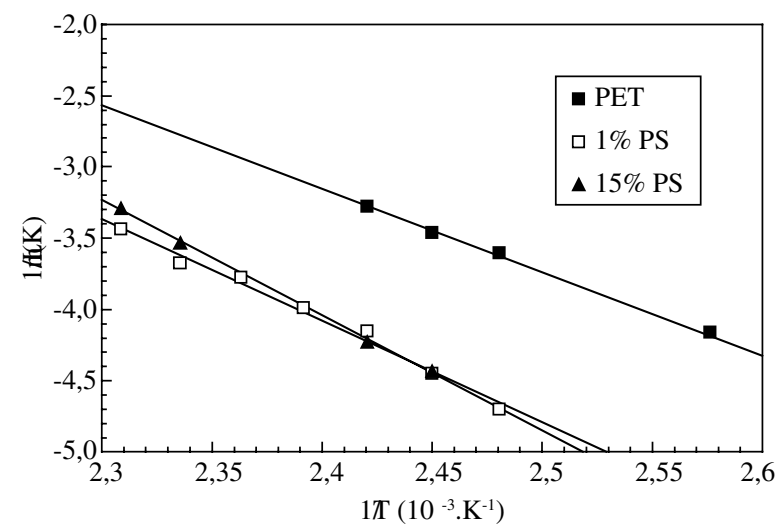

Figura 10. Curvas de Arrhenius para determinação da energia de ativação da cristalização isotérmica a frio do PET e das blendas com $1 \%$ e $15 \%$ de PS. segundo componente, de uma limitada solubilidade entre os componentes da blenda, de um aumento da energia de ativação para a cristalização isotérmica e de uma diminuição da cristalizabilidade do PET. Porém, nestas blendas a concentração do segundo componente variou entre $10-50 \%$. O que torna os resultados do presente trabalho muito interessantes é que a velocidade de cristalização do PET apresentou considerável diminuição com a adição de apenas 1\% de PS.

\section{Energia de ativação para a cristalização isotérmica}

A constante de velocidade $\mathrm{K}$ pode ser usada para determinar a energia de ativação da cristalização $(\Delta \mathrm{E})$ em condições isotérmicas. Assumindo que a cristalização é termicamente ativada, o parâmetro K pode ser escrito na forma de Arrhenius e a energia de ativação da cristalização pode ser obtida através do gráfico de $1 / \mathrm{n}$ Ln K versus $1 / \mathrm{T}_{\mathrm{c}}$ (Figura 10). A inclinação da curva determina $\Delta \mathrm{E} / \mathrm{R}$, onde $\mathrm{R}$ é a constante dos gases ${ }^{[32-33]}$. O valor calculado para a energia de ativação da cristalização primária do PET foi $48,70 \mathrm{~kJ} \cdot \mathrm{mol}^{-1}$, enquanto que as blendas com 1 e $15 \%$ de PS apresentaram valores de

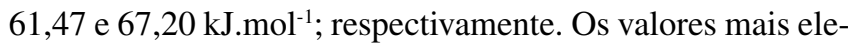
vados de energia de ativação para a cristalização das blendas em comparação com a do PET puro resulta da dificuldade de cristalização do PET na presença de poliestireno. Assim uma maior quantidade de energia deve ser fornecida para que

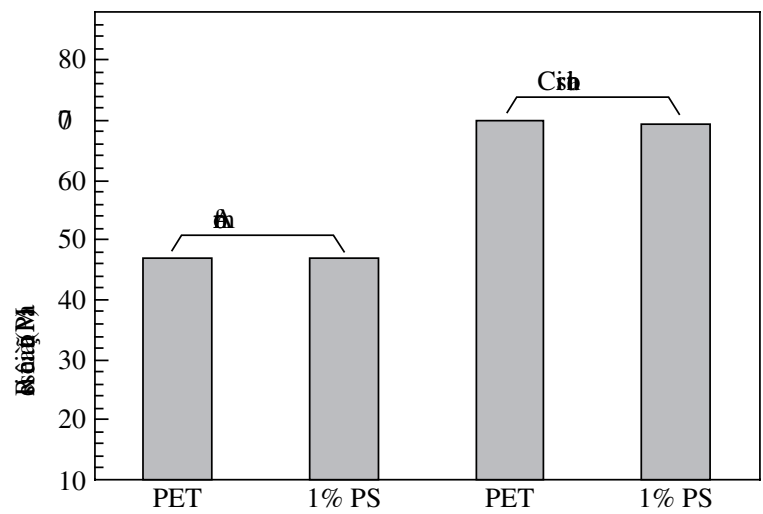

(a)

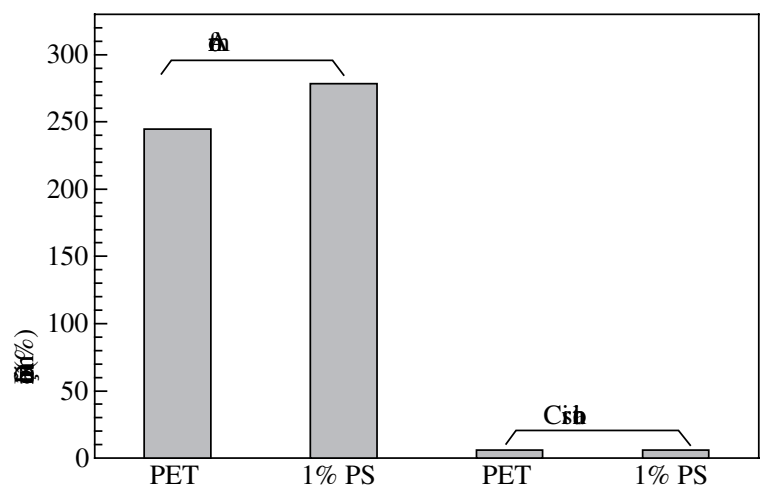

(b)

Figura 11. Resistência à tração e deformação máxima do PS, do PET e da blenda com $1 \%$ de PS para amostras amorfas e cristalinas. 

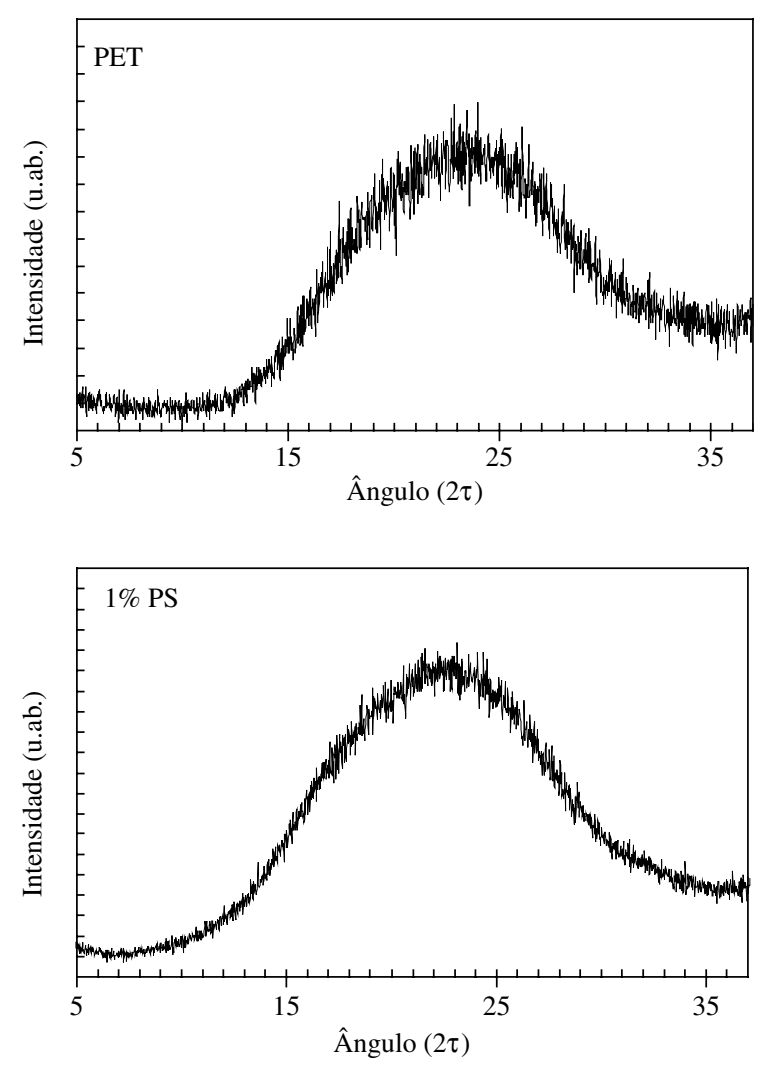

(a)
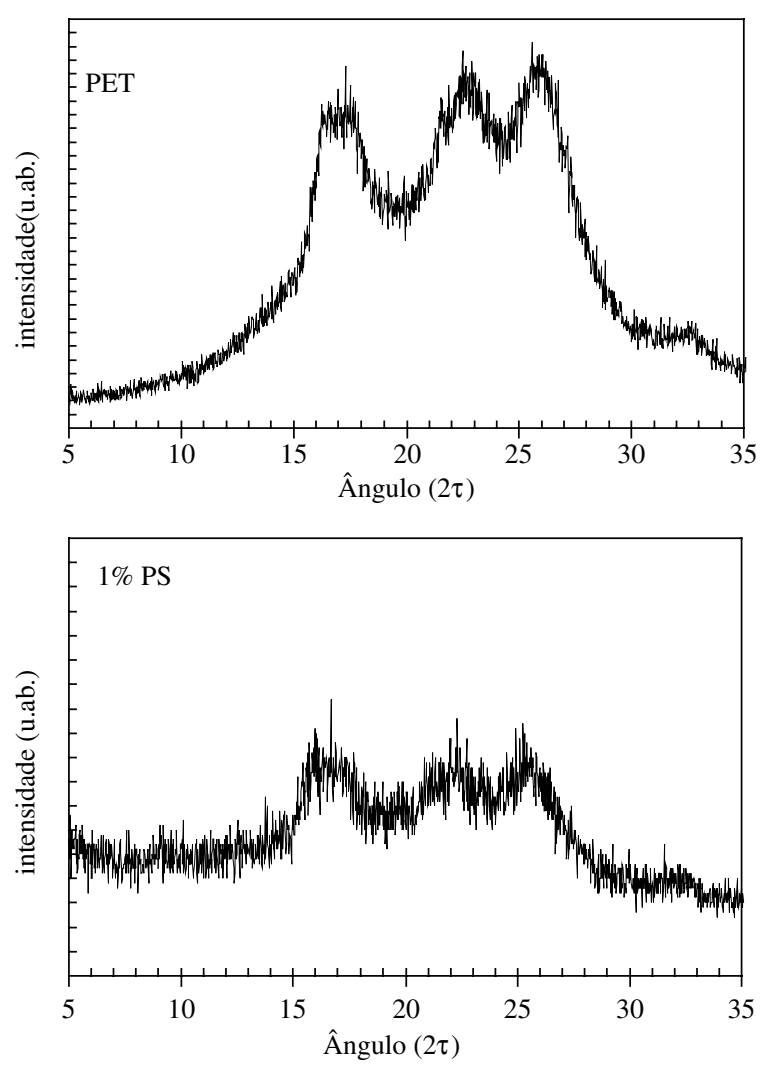

(b)

Figura 12. Difratogramas de raios $X$ do PET e da blenda com $1 \%$ de PS. a) Logo após processamento; e b) após cristalização por 1 hora a $150{ }^{\circ} \mathrm{C}$.

o processo de cristalização possa se desenvolver, o que está consistente com os resultados anteriormente mostrados.

\section{Propriedades mecânicas}

A Figura 11 apresenta os resultados obtidos para a resistência à tração do PET e da blenda com $1 \%$ de poliestireno no estado amorfo e no estado cristalino. Como descrito anteriormente, o material cristalino foi obtido por tratamento térmico dos corpos de prova injetados em molde gelado. A Figura 12a mostra os difratogramas de raios $\mathrm{X}$ dos corpos de prova originais (amorfos) do PET e da blenda com $1 \%$ de PS. Nota-se a ocorrência de apenas uma banda larga, sem picos bem definidos, característica de polímeros não cristalinos. Por outro lado, as amostras cristalizadas após a injeção (Figura 12b) apresentam picos de difração referente à ordenação cristalina nas duas composições analisadas. Nota-se na Figura 11 que a resistência à tração apresentou valores mais elevados nas amostras cristalizadas, pois a fase cristalina possui um maior empacotamento em relação à fase amorfa, o que dificulta os mecanismos de deformação molecular na região de comportamento elástico. Os resultados obtidos de deformação na ruptura também estão apresentados na Figura 11, mostrando que as amostras não cristalinas apresentaram valores mais elevados desta propriedade como conseqüência também do menor empacotamento molecular. Com relação à adição de poliestireno ao PET, nota-se que nas duas propriedades avaliadas o PET manteve o seu comportamento mecânico na presença do PS. Em vista dos resultados obtidos de cinética de cristalização, essa observação assume uma grande importância prática uma vez que o poliestireno retarda a cristalização a frio do PET sem interferir no seu desempenho mecânico.

\section{Conclusões}

Neste estudo a cinética de cristalização a frio em condições isotérmicas do PET e das blendas PET/poliestireno foi analisado através de calorimetria exploratória diferencial utilizando o tratamento de Avrami. As principais conclusões estão resumidas a seguir:

- As blendas poliméricas PET/PS formam misturas predominantemente bifásicas como observado por microscopia eletrônica de varredura e por análise dinâmico-mecânica. A depressão da temperatura de fusão de equilíbrio do PET com a presença de PS, por outro lado, indicou a ocorrência de uma limitada solubilidade entre os polímeros;

- A presença do PS em baixas concentrações (1\%) retardou significativamente a taxa de cristalização a frio do PET. O expoente de Avrami $n$ apresentou valores próximos a dois para o PET e para as blendas com PS; 
- A energia de ativação para a cristalização isotérmica do PET apresentou valores mais elevados nas blendas com PS, como resultado de um processo de cristalização mais dificultado; e

- As propriedades mecânicas do PET não sofreram alterações na blenda com $1 \%$ de PS. Isso ocorreu tanto para as blendas amorfas quanto para as cristalinas.

\section{Agradecimentos}

Os autores agradecem à Prof ${ }^{a}$ Maria Isabel Felisberti (Unicamp) pelas análises de DMA, à Rhodia (atualmente MG) pelo PET e à CAPES pela bolsa de doutorado.

\section{Referências Bibliográficas}

1. Groeninckx, G.; Reynaers, H.; Berghmans, H. \& Smerts, G. - J. Polym. Sci., Polym. Phys. Edn. 18, p.1311 (1980).

2. Pingping, Z. \& Dezhu, M. - Eur. Polym. J. 36, p.2471 (2000).

3. Zhao, J.; Yang, J; Song, R.; Linghu, X. \& Fan, Q. - Eur. Polym. J. 38, p.645 (2002).

4. Lim, J. Y. and Kim, S. Y - J. Appl. Polym. Sci. 71, p.1283 (1999).

5. Kint, D. P. R.; Rudé, E.; Llorens, J. \& Muñoz-Guerra, S. - Polymer 43, p.7529 (2002).

6. Van Antwerpen, F. \& Van Krevelen, D. W. - J. Polym. Sci, Polym. Phys. Edn. 10, p.2423 (1972).

7. Jabarin, S. A. - J. Appl. Polym. Sci. 34, p.85 (1987).

8. Kong, Y. \& Hay, J. N. - Polymer 43, p.1805 (2002).

9. Pó, R.; Occhiello, E.; Giannotta, G.; Pelosini, L. \& Abis, L. - Polym. Adv. Tech. 7, p.365 (1996).

10. Wellen, R. M. R. \& Rabello, M. S. - J. Mater. Sci. 40, p.6099 (2005).

11. Fann, D. M.; Huang, S. K. \& Lee, J. Y. - Polym. Eng. Sci. 38, p.265 (1998).

12. Maa, C. T. \& Chang, F. C. - J. Appl. Polym. Sci. 40, p.913 (1993).

13. Lee, J. S.; Park, K. Y.; Yoo, D. J. \& Suh, K. D. - J. Polym. Sci., Part B: Polym. Phys. 38, p.1396 (2000).
14. Ballara, A; Verdu, J. - Polym. Degrad. Stab. 26, p.361 (1989).

15. Grenier, D. \& Prud'homme, R. E. - J. Polym. Sci., Part B: Polym. Phys. Ed. 18, p.1655 (1980).

16. Olabisi, O.; Robeson, L. M. \& Shaw, M. T. - "PolymerPolymer Miscibility", Academic Press, New York (1979).

17. Cimmino, S.; Di Lorenzo, M. L. \& Silvestre, C. - Thermochimica Acta 321, p. 99 (1998).

18. Utracki, L. A. - "Polymer Alloys and Blends - Thermodynamics and Rheology", Hanser Publishers, Munich, p.55 (1989).

19. Hoffman, J. D. \& Weeks, J. J. - J. Research Natl. Bur. Standards 66-A, p.13 (1962).

20. Keith, H. D. \& Padden Jr, F. J. - J. Appl. Phys. 35, p.1270 (1964).

21. Keith, H. D. \& Padden Jr, F. J. - J. Appl. Phys. 35, p.1286 (1964).

22. Martuscelli, E.; Silvestre, C. \& Abate, G. - Polymer 23, p.229 (1982)

23. Calvert, P. D. \& Ryan, T. G. - Polymer 25, p.921 (1984).

24. Price, F. P. - J. Polym. Sci. Part A3, p.3079 (1965).

25. Avrami, M. J. - Chem. Phys. 7, p.1103 (1939).

26. Avrami, M. J. - Chem. Phys. 8, p.212 (1940).

27. Avrami, M. J. - Chem. Phys. 9, p.177 (1941).

28. Rabello, M. S. \& White, J. R. - Polymer 38, p.6379 (1997).

29. Rabello, M. S.; White, J. R. - Polymer 38, p.6389 (1997).

30. Jang, J.; Sim, K. - Polymer 38, p.4043 (1997).

31. Rahman, M. H. \& A. K. Nandi. Polymer 43, p.6863 (2002).

32. Cebe, P. \& Hong, S. - Polymer 27, p.1183 (1986).

33. Run, M.; Yao, C. \& Wang, Y. - Eur. Polym. J. 42, p.655 (2006).

Enviado: 20/09/06

Reenviado: 13/02/07

Aceito: $15 / 02 / 07$ 